\title{
Insect antifreezes come in from the cold
}

\section{Choy Hew}

The binding of ice crystals and inhibition of ice crystal formation are two unique properties of antifreeze proteins ${ }^{1}$. Although these proteins share a common function in conferring freeze resistance, they are suprisingly diverse in structure. Cold marine fishes produce at least four different types of antifreeze glycoproteins (AFGPs) and antifreeze proteins (AFPs), and coldtolerant plants, such as winter rye, produce anti-freeze proteins that resemble pathogenesis-related proteins ${ }^{2}$. Until now, however, the nature of antifreeze proteins from insects (although implicated in several earlier reports) has not been elucidated. Two new papers by Davies, Walker and coworkersone in this issue reporting the characterization of the spruce budworm (Choristoneura fumiferana) $\mathrm{AFP}^{3}$ and the other describing the AFP from the common mealworm beetle (Tenebrio molitor $)^{4}$-therefore represent major advances in insect biology. At the same time, they raise questions about the mode of action of these superior antifreeze proteins and their evolution.

Spruce budworm larvae hatch from egg masses in the summer and hibernaculae (silk tents) in the winter as second instar larvae without having eaten. During winter months, they can be exposed to temperatures approaching $-30^{\circ} \mathrm{C}$, but still resist freezing. In the spring, they complete development through the other instars while eating pine needles, pupate, and then turn into moths. The production of the antifreeze proteins is, therefore, an integral part of their survival strategy (Fig. 1).

Considering the extreme conditions of their environment, it is not surprising that these insects have evolved AFPs that are the most active antifreezes known. At a concentration of $1 \mathrm{mg} / \mathrm{ml}$, they cause a freezing point depression of $4.0-5.5^{\circ} \mathrm{C}$ degrees, which is fourfold higher than that of fish AFPs attained at concentrations of $10-20 \mathrm{mg} / \mathrm{ml}$. At lower concentrations, the insect AFPs are 10-100-fold more active.

Choy Hew is a senior scientist at the Hospital for Sick Children, Toronto, and professor, departments of laboratory medicine \& pathobiology and biochemistry, University of Toronto, Banting Institute, 100 College Street, Room 351, Toronto, Ontario Canada M5G $1 L 5$ (choy.hew@utoronto.ca).
How these unprecedented activities are accomplished is unknown at present. An examination of ice crystal morphology indicates that insect AFPs have a different effect on ice crystal growth, but these morphological studies shed little light on mechanism of action. Presumably, detailed structural elucidation will be needed to verify whether such insect AFPs contain multiple ice binding motifs like type I fish AFPs ${ }^{5}$, flat amphipathic

\section{IMAGE UNAVAILABLE FOR COPYRIGHT REASONS}

structural relatedness between budworm and beetle AFPs, and suggest that they both arose by a convergent evolution mechanism. The AFGPs of antarctic fishes and AFPs of type II fish have been postulated to be derived from trypsinogen and carbohydrate-binding domains of lectins ${ }^{10}$, respectively. As yet, the evolution of the type I and type III AFPs is unknown. The two insect AFPs described by Walker and colleagues do not share any structural homology with any known AFPs nor with any other proteins in GenBank (Bethesda, MD). These proteins, therefore, are new members of the AFP superfamily. Presumably, adaptation to the freezing environment is such a common and vital phenomenon that different organisms may have developed or evolved distinct mechanisms of action to inhibit ice crystal formation.

How to exploit some of the exceptional properties of these proteins? First, with the availability of the AFP gene sequence, it might be feasible to inhibit mealworm or budworm AFP genes using antisense, ribozyme, or other approaches and thereby prevent

ice-binding sites like type III fish $\mathrm{AFPs}^{6}$, or other novel ice-binding conformations.

Both the spruce budworm and the mealworm beetle AFPs are rich in serine, threonine, glycine, and cysteine. They contain several 12 amino acid repeats of Cys'ThrXXSerCysXXAlaXThr (three-letter amino acid code, where $\mathrm{X}$ is any residue), with variants differing in the number of the repeats. It seems likely that this repeat region forms a functional domain along the lines of the 11 amino acid repeat of ThrXXAsp/AsnXXXXXXX (where X is predominantly alanine) observed in the type I AFPs from the winter flounder and other righteye flounders.

In these helical AFPs, the threonine at each repeat has a length of $16.5 \AA$ and matches the ice lattice along a pyramidal plane ${ }^{i, 7}$. It is feasible that the formation of the disulfide bond within each repeat in the beetle AFPs may strengthen their structural rigidity and enhance ice binding in a manner similar to the salt bridges in type I

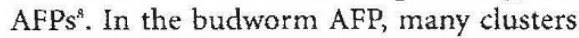
of threonine and serine are seen; mutagenesis of these residues, together with structural studies, should prove useful in probing function.

These authors' work reinforces the notions of widespread occurrence and structural diversity across the antifreeze protein family. Except for a similarity in molecular mass $(\sim 9 \mathrm{kDa})$, Walker et al. do not find any pest infestation of pine forest. On a more general front, in addition to their ice-binding properties and the inhibition of ice recrystallization, AFPs/AFGPs have been shown to protect the leakage of ions in liposomes ${ }^{11}$ and human platelets from cold storage $^{12}$. It seems likely that the vastly superior insect AFPs may find a wider application in the cryoprotection of cells and tissues. In the food industry, where AFPs have been used to inhibit ice recrystallization in frozen foods, a more active AFP might also be more economical as a food ingredient. In the latter scenario, however, "icy" consumer resistance might be a new hurdle for these insect-derived antifreezes to overcome.

1. Hew, C.L. and Yang, D.S. 1992. Eur. J. Biochem 203:33-42.

2. Hon, W.C., et al. 1995. Plant Physiol. 104:971-980.

3. Tyshenko, M.G., et al. 1997. Nature Biotechnology 15:887-890.

4. Graham, L. 1997. Nature, August 21, in press.

5. Sicheri, F. and Yang, D.S.1995. Nature 375:427-431

6. Jia, Z., et al. 1996. Nature 384:285-288.

7. Wen, D. and Larsen, R.A. 1992. Biophys. J. 63:1659-1662

8. Chakrabartty, A and Hew, C.L. 1991. Eur. J. Biochem. 202:1057-1063.

9. Chen, L., et al, 1997. Proc. Natl Acad. Sci. USA 94:3811-3818.

10. Ewart, K.V., et al. 1996. J. Biol. Chem 271:16627-16632

11. Hays, L.M., et al. 1996. Proc. Natt. Acad. Sci. USA. 93:6835-6840.

12. Tablin, F., et al. 1996. J. Cellular Physiol. 168:305-313. 